\title{
Colorectal lymphoma simulating inflammatory colitis and diagnosed by immunohistochemistry
}

\author{
S. Sagar ${ }^{1}$, P. Selby ${ }^{1}$, J. Sloane ${ }^{2}$ and T.J. McElwain ${ }^{1}$ \\ Departments of ${ }^{1}$ Medicine and ${ }^{2}$ Pathology, Royal Marsden Hospital, Downs Road, Sutton, Surrey SM2 5PT, UK.
}

\begin{abstract}
Summary: Lymphomatous colitis is probably an uncommon syndrome but its recognition may be of importance to patients under the care of gastroenterologists or oncologists. The case of an elderly man with lymphocytic lymphoma who developed lymphomatous colitis 6 years after initial presentation is reported.
\end{abstract}

\section{Introduction}

Lymphoma uncommonly involves the large bowel (Lewis et al., 1978) but lymphomatous infiltration has been occasionally reported diffusely to involve the large bowel and simulate inflammatory bowel disease (Renton \& Blackshaw, 1976; Friedman et al., 1968; Weir et al., 1980). This may present substantial diagnostic difficulty (Friedman et al., 1968; Weir et al., 1980). We report here a case of large bowel lymphoma simulating an inflammatory bowel disorder with some features of both ulcerative and Crohn's proctocolitis which was conclusively diagnosed by immunocytochemical methods.

\section{Case report}

In 1977 a 66 year old man presented with wide-spread lymphadenopathy and a lymph node biopsy revealed a well differentiated lymphocytic lymphoma (Rappaport Classification). He was treated with chemotherapy and entered a remission but subsequently relapsed. Further courses of chemotherapy and radiotherapy to local areas of lymphadenopathy resulted in partial control of his disease for 6 years. During this time lymphomatous infiltration of the bone marrow was detected.

In 1983 he developed severe watery diarrhoea up to 15 times daily with no obvious bleeding. Examination revealed generalized bulky lymphadenopathy and abdominal tenderness. Proctosigmoidoscopy revealed a granular, hyperaemic mucosa which bled on contact. Scattered superficial ulcers were seen. A barium enema (Figure 1) revealed ulcerated mucosa in the rectum and sigmoid with similar but less severe changes

Correspondence: P. Selby, M.A., M.D., M.R.C.P.

Accepted: 16 April 1985

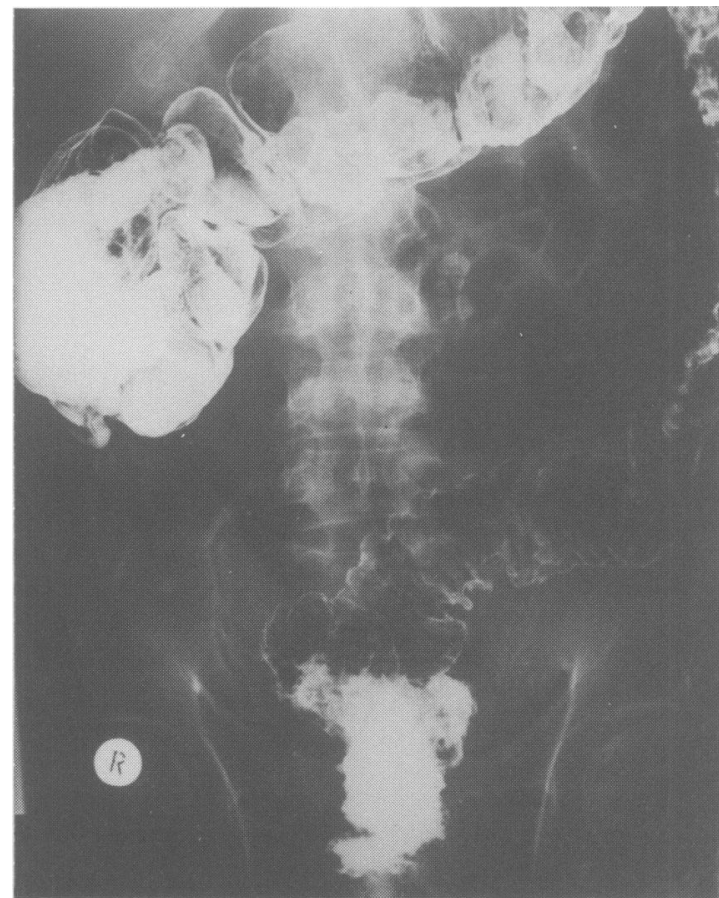

Figure 1 A barium enema examination revealing an abnormal mucosa with ulceration and in particular quite deeply penetrative ulceration in the region of the rectosigmoid.

extending to the splenic flexure of the colon. Although the disease appeared to be limited to the distal colon and rectum, suggesting an ulcerative colitis, some of the ulcers appeared very penetrative and were more suggestive of Crohn's disease. A rectal biopsy showed collections of pus on the luminal surface and small foci

(C) The Fellowship of Postgraduate Medicine, 1986 
of granulocytic infiltration of the surface and glandular epithelium. Most of the glands showed some mucin depletion but there was no significant glandular destruction. Very rare crypt abscesses were seen. The lymphoid follicles were enlarged up to $0.2 \mathrm{~cm}$ and sometimes exhibited irregular margins. They were composed almost entirely of morphologically mature lymphocytes with occasional small clusters of large lymphoid cells with vesicular nuclei and conspicuous nucleoli. Germinal centres were not seen, however. There was a moderate increase in plasma cells in the lamina propria but lymphocytes were present in normal numbers. Capillaries in the lamina propria were dilated. The appearances were regarded as consistent with a non-specific colitis. A clinical diagnosis of ulcerative colitis was made and was treated with local and then systemic corticosteroids.

After 3 weeks' treatment there was no improvement and a further sigmoidoscopy was performed. There was no change in the appearance of the rectum or sigmoid colon. A second barium enema was done which revealed marked deterioration compared with the previous appearances. A second rectal biopsy was undertaken. The conventional histological appearances were essentially similar to those in the first specimen, but one of the lymphoid aggregates was

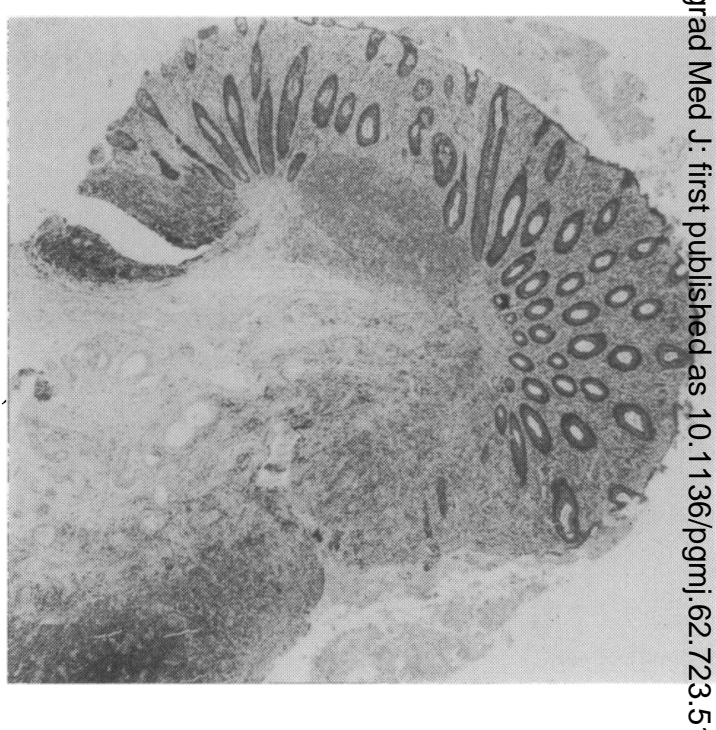

Figure 2 A second rectal biopsy showing mucin deple-tion but little glandular loss. One of the smaller circumscribed lymphoid nodules is present in the upper half of the field and the larger irregular mass with overlying ulcerad tion in the lower half. $(\mathrm{H} \& \mathrm{E} \times 35)$.
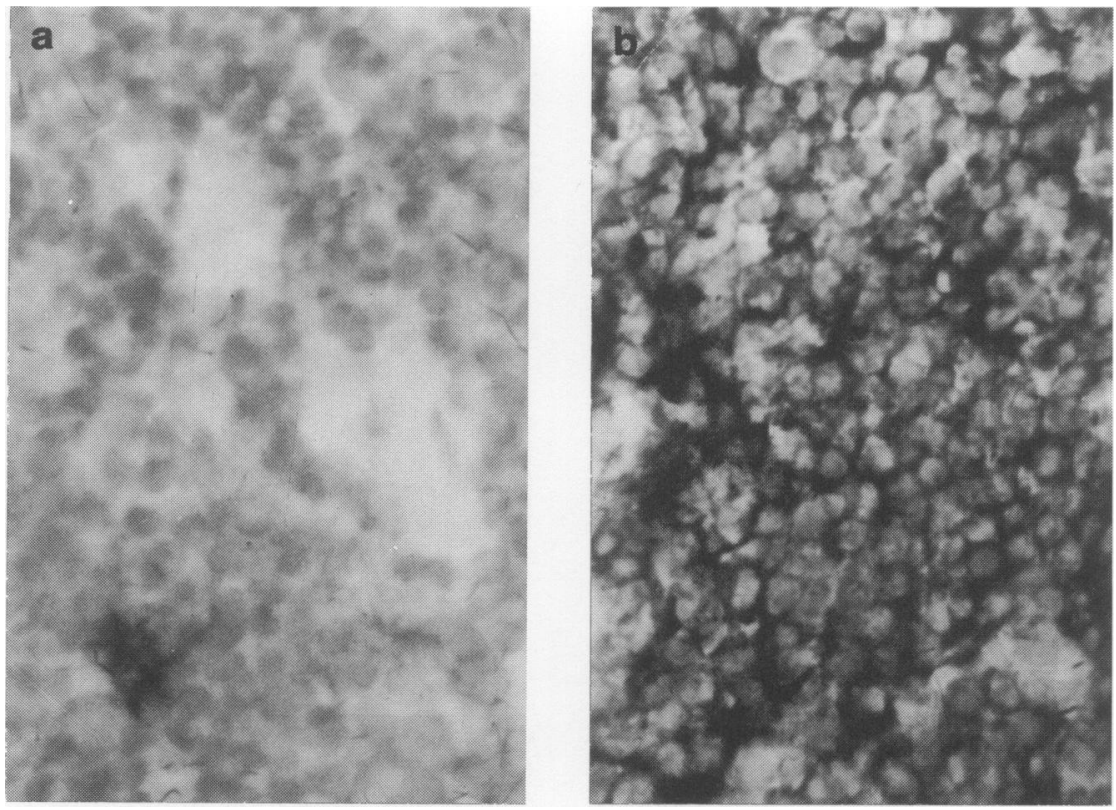

Figure 3 Immunohistological staining of one of the smaller nodules for (a) lambda and (b) kappa immunoglobulin $\stackrel{?}{+}$ light chains. The lymphocyte membranes are negative for lambda but there is a solitary positive plasma cell in the $\square$ bottom left of the figure. $(\times 504)$. 
much larger and associated with ulceration of the overlying mucosa (Figure 2). Half the specimen was snap frozen in liquid nitrogen for immunohistological analysis with conventional antisera to immunoglobulin light and heavy chains. A double layer technique using an alkaline phosphatase conjugated second antibody was employed. The cell membranes of the lymphocytes of all the lymphoid follicles were positive exclusively for kappa light chains (Figure 3) and mu heavy chains and were negative for lambda light and gamma, delta and alpha heavy chains. This indicated that they represented a monoclonal proliferation and that the follicles were, in fact, nodules of malignant lymphoma of the lymphocytic type. The monoclonal cells were not seen in the lamina propria nor in the submucosa away from the lymphoid nodules. Immunoglobulin staining also revealed the presence of numerous plasma cells; most of these were in the lamina propria but some were seen in the submucosa and a small number were also present in the lymphoid nodules. In all locations the plasma cells consisted of mixtures of kappa and lambda positive cells with a normal ratio of about 2:1. Most were $\operatorname{IgA}$ positive with a small number of IgM positive cells. The plasma cells were thus a normal polyclonal reactive population and not part of the lymphoma.

He received further systemic steroids and total abdominal irradiation but there was little improvement. He deteriorated and died a few weeks later. Post-mortem was not performed.

\section{Comment}

Severe and extensive ulcerative colitis is known to be associated with adenocarcinoma of the large bowel

\section{References}

FRIEDMAN, H.B., SILVER, G.M. \& BROWN, C.H. (1968). Lymphoma of the colon simulating ulcerative colitis. American Journal of Digestive Diseases, 13, 910.

LEWIS, K.J., RANCHOD, M. \& DORFMAN, R.R.F. (1978).

Lymphomas of the gastrointestinal tract. Cancer, 42, 693.

RENTON, P. \& BLACKSHAW, A.J. (1976). Colonic lymphoma but the association between inflammatory bowel disease and malignant lymphoma is less well recognized. Considerable uncertainty exists about the nature of the relationship between lymphomatous infiltration of the large bowel and inflammatory colitis. Several cases have been described in which localized, or occasionally multicentric, lymphoma of the large bowel have developed in patients after many years of typical ulcerative colitis (Renton \& Blackshaw, 1976). A causative relationship of the colitis to the lymphoma has been suggested. On the other hand, there have been several reports of patients with infiltration of the large bowel who were diagnosed as having either Crohn's disease (Weir et al., 1980) or ulcerative colitis (Friedman et al., 1968) until a generalized lymphoma developed and the diagnosis of lymphomatous infiltration was made.

The present case illustrates two important points. Firstly, it is clear that the colitic syndrome developed as a result of a lymphomatous process which had been established in the patient for 6 years and there is no evidence for pre-existing inflammatory bowel disease. Secondly, modern methods of immunohistochemistry have allowed a diagnosis to be made with certainty. The diagnosis had proved difficult in this case using morphology alone and this difficulty has been a feature of previous reports (Friedman et al., 1968). Immunological characterization of the cellular infiltrate is a powerful tool which appears to be capable of resolving diagnostic difficulties in some of these cases.

Lymphomatous colitis is probably an uncommon syndrome but its recognition may be of importance to patients under the care of gastroenterologists or oncologists. Increasing awareness of the condition and improved methods of its diagnosis might show that it is not as uncommon as has been previously thought.

complicating ulcerative colitis. British Journal of Surgery, 63, 542.

WEIR, A.B., POON, M.C., GROARKE, J.F. \& WILKERSON, J.A. (1980). Lymphoma simulating Crohn's colitis. Digestive Diseases and Sciences, 25, 69. 